\title{
Triton: Fascinating Moon, Likely Ocean World, Compelling Destination!
}

\author{
Candice J. Hansen \\ 626-483-5640 \\ Planetary Science Institute \\ cjhansen@psi.edu
}

\section{Co-authors:}

Will Grundy, NAU

Jason Hofgartner, JPL

Emily Martin, SI

Karl Mitchell, JPL

Francis Nimmo, UCSC

Carol Paty, UOregon

James Roberts, JHU/APL

Kirby Runyon, JHU/APL

Lynnae C. Quick, GSFC

Paul Schenk, LPI

Alan Stern, SwRI

Orkan Umurhan,

SETI/NASA

\section{Co-signers:}

Patricio Becerra

Ross Beyer

Alyssa Rhoden

James Keane

Bonnie Burrati

Walt Harris

Rebecca Schindhelm

Anne Verbiscer

Simon Porter

Chloe Beddingfield

Marc Neveu

Alex Patthoff

Kathy Mandt

Sierra Ferguson

Raluca Rufu

Vishaal Singh
Erin Leonard

Amanda

Hendrix

K.-Michael Aye

Ganna Portyankina

Tom Nordheim

Terry Hurford

Richard Cartwright

Mallory Kinczyk

Zach Ulibarri

Julie Castillo-Rogez

Alex Hayes

Carly Howett

Rosaly Lopes

Kurt Retherford

Chuanfei Dong

Cesare Grava

Steve Vance

Michael Poston

Krishan Khurana

Andrew Annex

Jorge Núñez

Mark Sykes

Paolo Tortora

Adam Masters

Ian Cohen

Louise Prockter

H. Todd Smith

Tom Stallard

Leigh Fletcher

Yohai Kaspi

Alessandro Migliorini

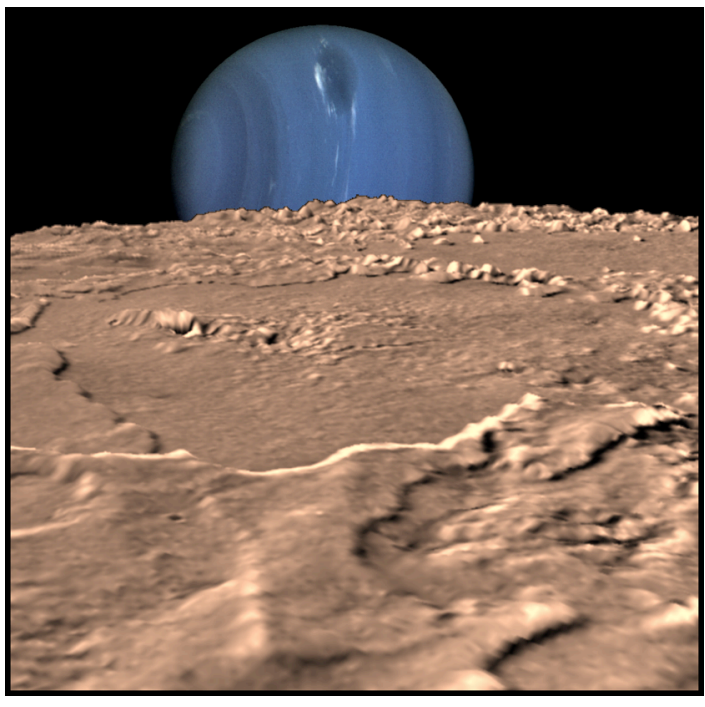

Corey Cochran

Sarah Moran

Jennifer Scully

Geoffrey Collins

Tilmann Denk

Alice Lucchetti

Maurizio Pajola

Sarah Fagents

Benoit Noyelles

Robert Marcialis

Carly Howett

Marzia Parisi

Tracy Becker

Imke de Pater

Cynthia Dinwiddie

Bryan Holler

Michelle Kirchoff

Mark Gurwell

Tanguy Bertrand

Gianrico Filacchione

Federico Tosi

Adrienn Luspay-Kuti

Racine Swick

Randy Kirk

Alfred McEwen

Abigail Rymer

Krista Soderlund

Linda Spilker

Sebastian Rodriguez 


\section{Triton: Fascinating Moon, Likely Ocean World, Compelling Destination!}

\section{Why Explore Triton?}

Neptune's moon Triton has been explored by just one spacecraft, Voyager 2, in 1989. Images revealed a unique geologically young surface with landforms found nowhere else in the solar system [1]. Geysers erupt from a surface with a temperature of just 38K [2]. A tenuous nitrogen atmosphere is in vapor pressure equilibrium with its surface ices, similar to Pluto [3, 4, 5]. Triton is also noteworthy for its retrograde, highly inclined orbit, making it almost certainly a captured Kuiper Belt dwarf planet, with a differentiated interior heated by tidal braking [6, 7]. Triton, nearly a twin in size to Pluto, provides a unique window into evolutionary scenarios for a dwarf planet tidally-activated by a giant planet. Triton is thus a window into, and an opportunity for, the next stage in Kuiper Belt dwarf planet exploration.

Earth-based spectroscopy shows the presence of $\mathrm{H}_{2} \mathrm{O}$ and $\mathrm{CO}_{2}$, which are presumed to form the surface bedrock $[8,9]$. Volatile ices $\mathrm{N}_{2}, \mathrm{CO}$, and $\mathrm{CH}_{4}$ are present and expected to migrate across the surface seasonally [10]. Many hypotheses for Triton's unique geology invoke the behavior of volatiles, however Voyager did not carry a spectrometer capable of determining Triton's surface composition. Surface composition of Triton and potential linkage to ocean chemistry represents a critical gap in our understanding of this outer solar system moon.

Is Triton an ocean world? Crater counts suggest that Triton's surface age is $<100 \mathrm{Ma}$, possibly $<10 \mathrm{Ma}$ old $[11,12]$. Strong tidal heating anticipated from an ancient capture event would not explain the currently observed young surface age, while more recent capture is unlikely. Although Triton's orbit has long since circularized and eccentricity tides are negligible today, obliquity tides could be particularly strong on Triton because of its high inclination. These tides could supply the necessary energy for surface processes that would erase craters, and possibly maintain a liquid layer below the surface [13].

Do the oceans of moons of outer planets provide habitable environments and host life? The strategic path to answer this question led to OPAG's Roadmap to Ocean Worlds endorsement: Among the candidate ocean worlds in the solar system "Triton is deemed the highest priority target to address as part of an Ocean Worlds Program” [14]. Liquid water, organic compounds, and chemical energy are generally accepted to be necessary ingredients for life. Telescopic observations of Triton's surface and atmosphere reveal abundant elemental building blocks, especially carbon, hydrogen, oxygen and nitrogen ( $\mathrm{CHON})$, and suggest ionospheric production of organic compounds, which by analogy with Titan and Pluto would include materials of high chemical potential. Furthermore, Voyager 2 images provide tantalizing hints of exchange processes between Triton's surface and subsurface. Confirmation of a rich organic-chemistry environment and ice-shell transport processes connecting Triton's proposed ocean to its surface would place Triton among the highest value targets in the search for life, adding to the diversity of potentially habitable worlds, all the way out at $30 \mathrm{AU}$.

In the following pages we review the outstanding science questions, regarding Triton itself, its interaction with its ice giant planet, and where it fits in the search for life. Voyager provided us with a snapshot in time, high resolution data on just one quarter of the surface: a teaser of the scientific riches still to be explored.

A mission to Triton is guaranteed to fill fundamental knowledge gaps in multiple high priority areas for the next decade of exploration. 


\section{Triton's Geology and Surface Composition - Topography shaped by Cryovolcanism?}

Voyager flew by Triton at an altitude of $\sim 40,000 \mathrm{~km}$. Although global coverage of the illuminated terrain was achieved over Triton's 5.877 day rotation period, only the images of the Neptune-facing side south of $\sim 40^{\circ} \mathrm{N}$ achieved spatial resolution of $1-3 \mathrm{~km} /$ line pair. At the time of the Voyager flyby Triton was experiencing an extreme southern spring, with a sub-solar latitude of $45^{\circ} \mathrm{S}$, which meant much of the northern hemisphere was in polar night.

The highest resolution mosaic, shown in Figure 1, revealed exotic landforms unlike any on the moons of Jupiter, Saturn or Uranus. Cantaloupe terrain, walled plains, smooth plains, and guttae are among the many unique feature types imaged, as summarized in Croft et al., 1995 [15]. Even landforms such as double ridges, which have analogues on other moons such as Europa [16], have a different morphology on Triton: Triton's double ridges are an order of magnitude wider than Europa's and half as tall.

At the temperatures of the outer solar system water and $\mathrm{CO}_{2}$ ices will generally behave as bedrock. The likely presence of other volatiles such as $\mathrm{NH}_{3}$ and $\mathrm{CH}_{3} \mathrm{OH}$ in the mix leads to the intriguing possibility that Triton's geology shows archetypal examples of cryovolcanism and other exotic processes, perhaps similar to Pluto. ["Cryovolcanism encompasses processes involving the deformation, intrusion, and extrusion of liquids, slushes and "warm" plastically deforming solids composed of mixtures of low-melting point materials" [15].]

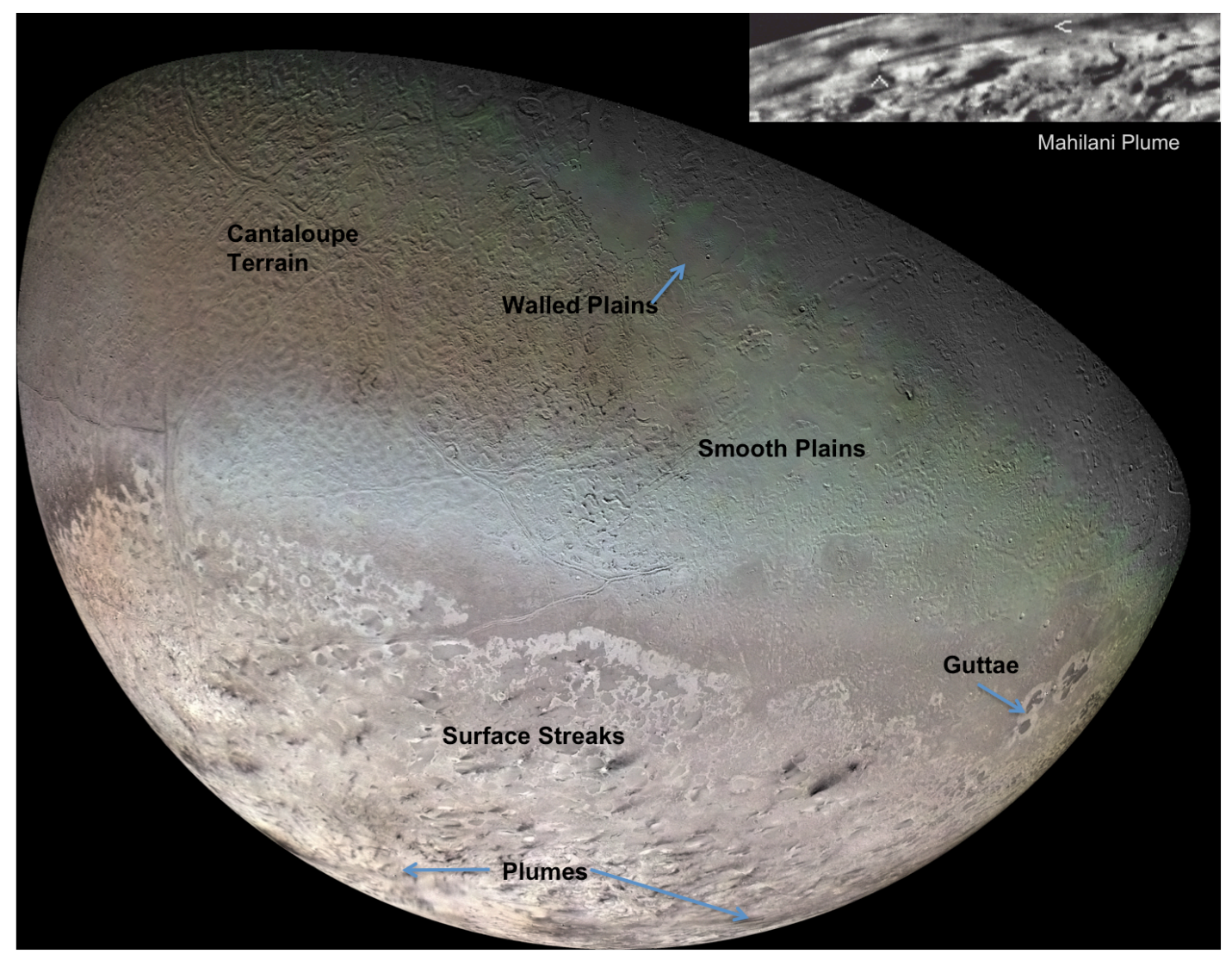

Figure 1. Many of Triton's unique landforms suggest the possibility of cryovolcanism.

Some of the simplest explanations for features like guttae or round and pitted structures within the smooth plains share characteristics with other volcanic structures observed elsewhere in the solar system, including Earth. While error bars on the absolute crater model ages for 
Triton are large, they tell the story of a young, dynamic surface heavily modified by endogenic geologic processes.

The location and orientation of ridges and other smaller scale tectonic structures may outline a tectonic pattern that could be used to elucidate the stress mechanisms acting upon Triton's icy shell. The long length of double ridges indicates that a global scale stress mechanism is most likely and may include diurnal tidal stress, obliquity stresses, nonsynchronous rotation, despinning, or orbital recession. Smaller tectonic structures near the smooth plains and guttae require higher-resolution imagery with near-global coverage to determine how widespread they are, which will provide insight into whether these structures are driven by global or regional stresses.

While morphology is important, composition is a key test of formation hypotheses for Triton's landforms. The compositions of Triton's individual surface units are unknown because Voyager did not have an instrument that could map surface composition, and Earth-based / HST observations have very limited spatial resolution. Even next generation 30-m class telescopes would not obtain resolutions better than $\sim 100-200 \mathrm{~km}$, thus leaving many unanswered questions pertaining to the compositions and formation of many of Triton's enigmatic surface features. Examples include:

- Do guttae result from sublimation erosion or surface collapse over hot spots?

- Is cantaloupe terrain formed by diapirism?

- Are walled plains formed by the eruption of cryomagma?

How does the interplay of tidal dissipation, heat transfer, tectonics, cryovolcanism, diapirism, and surface-atmosphere interactions drive resurfacing on Triton? The possibility that Triton's exotic landforms are formed by cryovolcanic processes can only be determined by a new mission carrying a capable imaging spectrometer.

\section{Triton's Volatiles: Winds, Seasonal Processes and Climate}

Triton's atmosphere is $\mathrm{N}_{2}$-dominated with traces of $\mathrm{CH}_{4}, \mathrm{CO}$, and other species, and hazes, similar to Pluto [17]. Similar to Mars, which has a $\mathrm{CO}_{2}$ atmosphere in vapor pressure equilibrium with $\mathrm{CO}_{2}$ surface ice, Triton's $1.4 \mathrm{~Pa}$ (in 1989) nitrogen atmosphere is expected to sublimate and condense seasonally, forming seasonal polar caps [18, 19]. Polar ice sublimation drives winds, as evidenced by the orientation of fan-shaped deposits on Triton's surface and the orientation of Triton's geyser-like plumes [20,21]. Geological evidence for winds may parallel those of Pluto: wind streaks and dune-like landforms [22]; finding such evidence on Triton requires imaging at better than a few hundred meter resolution.

Changes in Triton's atmospheric pressure since the Voyager flyby have been detected in stellar occultations observed from Earth [e.g. 22]. Insolation, which drives surface ice energy balance with the atmosphere, and is controlled by the subsolar latitude, has a complex $>600$ year cycle due to the convolution of Triton's inclined orbit with Neptune's obliquity [23].

$\mathrm{CO}$ and $\mathrm{CH}_{4}$ are minor constituents in the atmosphere and in surface ices, however, as observed on Pluto, these species also move seasonally and have interesting effects on the surface. Triton's bright southern hemisphere may have shown evidence of the distribution of seasonally mobile ice during the Voyager 2 flyby. A timely return to Triton with an imaging spectrometer could easily test this hypothesis.

Earth-based data from the decades since the Voyager flyby is consistent with $\mathrm{N}_{2}$ sublimating away from the south pole, leaving longitudinally unchanging $\mathrm{H}_{2} \mathrm{O}$ and $\mathrm{CO}_{2}$, 
presumably the bedrock in the southern hemisphere $[8,9,24] . \mathrm{N}_{2}$ and $\mathrm{CO}$ ices are detected spatially concentrated in the sub-Neptune hemisphere, while the maximum $\mathrm{CH}_{4}$ absorption is $300^{\circ} \mathrm{E}$ longitude [9].

- Where are Triton's volatiles located on its surface?

- What drives their distribution? Are the ratitudinal color bands imaged by Voyager consistent with frost on the move seasonally?

- Are there permanent $\mathrm{N}_{2}$ polar caps at both poles? Or conversely just one in the southern hemisphere [25]?

- Since the time of the Voyager flyby the subsolar point has passed through the southern summer solstice and is approaching the equator. How much mass has been transferred into the northern polar region? What is the escape rate?

A mission to Triton that maps surface ices and measures the density of the atmosphere will illuminate Triton's complicated climate history.

\section{Triton's geysers}

A completely astounding result from Voyager 2 was the discovery of at least 2 plumes erupting from the surface [26]. These were hypothesized to be solar-powered geysers: driven by seasonal sublimation and storage of nitrogen gas under translucent ice, pressurized, and explosively released [27]. [The Voyager team noted the unorthodox use of the term "geyser" compared to Earth's geysers.] The numerous dark fans on the bright surface with orientations determined by the prevailing wind were likely deposited on the surface by eruptions that were no longer active at the time images were taken by Voyager. The model of solar-powered activity has challenges however, including the large size of the sub-ice nitrogen reservoir required, and thus the origin of the plumes is still a mystery. New datasets for the solar-driven jets of Mars and the endogenic plumes of Enceladus lead us to re-examine the solar-driven hypothesis and ask how likely such plumes are to be connected with a subsurface liquid water ocean [28].

- Are Triton's plumes solar-driven or endogenic?

- What do the sites and timings of plume occurrence tell us about the energetics and relevant processes?

- What volatile reservoir do they access? Can the origin of the plumes be traced back to fluid pockets that reside at shallow levels in Triton's icy crust? Or do they tap the subsurface ocean?

If endogenic, the plumes may be sampling a subsurface reservoir, possibly a subsurface ocean, enabling access to ascertain its habitability.

\section{Triton's Unique Ionosphere and Interaction with Neptune's magnetosphere}

Voyager radio science observations revealed a significant ionosphere with a well-defined peak at $\sim 350 \mathrm{~km}$ altitude [4]. However, the distance and the geometry of the Triton closest approach precluded in situ observations of either the ionosphere or its interaction with Neptune's magnetosphere. Heavy ions, likely associated with Triton's exosphere, were observed to be concentrated towards Neptune's magnetic equator [29, 30].

The outer extent of the high-energy $(>\sim 1 \mathrm{MeV})$ radiation belt particles at Neptune is set by Triton's orbit, thought to possibly "sweep up" these particles as it orbits Neptune [31]. This behavior extends to lower energies, but electrons with energies of $10 \mathrm{~s}$ of $\mathrm{keV}$ are present at 
Triton's orbit and may be the principal driver for auroral emissions seen by Voyager. Changes in the electron spectral index ( $\sim 20$ to $60 \mathrm{keV})$ peaks at Triton's location and is an additional signature of the significant interaction of the moon with Neptune's magnetosphere at these energies [32, 33].

One or more earth occultations observed by a spacecraft can help quantify the temporally and spatially varying density and temperature of Triton's ionosphere. Passage through Triton's wake would further constrain composition by in situ measurements of material coming from Triton's ionosphere and exosphere. Sampling precipitating energetic particles local to Triton would serve to quantify the energy source responsible for a significant portion of the ionosphere's production and maintenance.

- How does a highly conducting Triton interact with the corotating magnetosphere of Neptune?

- Is this process related to the generation and maintenance of Triton's intense ionosphere, which has a peak electron density of $2-5 \times 10^{4} \mathrm{~cm}^{-3}$ ?

- How does Triton's intense ionosphere influence organic chemistry in its atmosphere?

Studying the plasma interaction between Triton and Neptune will give us new insights into the structure and chemistry of Triton's upper atmosphere and reveal how Triton loses material from its atmosphere to fill the magnetosphere of Neptune.

\section{Is Triton an Ocean World?}

Triton's bulk composition indicates a rock:ice mass ratio of $\sim 2$. Based on the extreme degree of tidal heating following capture, we expect that Triton has differentiated into a rocky core with a water ice mantle. Analysis of bodies with substantial rock content in their bulk composition led Hussman (2006) [34] to propose that formation of liquid layers on icy moons could be common from radiogenic heating of water laced with ammonia. Hammond (2018) [35] find that mixtures of ammonia and water can freeze and leave ammonia-rich ice near Triton's surface that would facilitate cryovolcanism and increase the likelihood of maintaining a

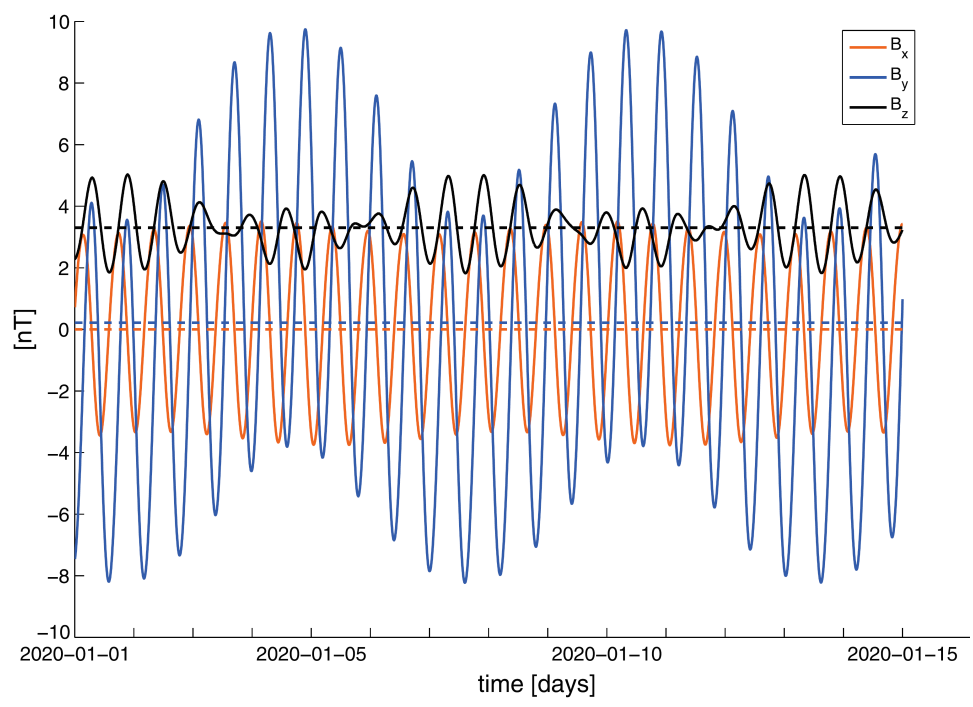
subsurface liquid ocean. Potentially cryovolcanic landforms on Triton's surface are intriguing indicators of resurfacing [15].

Figure 2. Neptune's magnetic field at the location of Triton varies strongly with time and possesses harmonics at both the synodic rotation period of Neptune and the orbital period of Triton. Such harmonics can be exploited for induction sounding of an ocean in Triton from a single or multiple flybys of Triton. Figure reproduced from Saur et al. (2010).

Because Neptune's magnetic dipole has a tilt of $47^{\circ}$ relative to its spin axis and the field also has strong quadrupolar and octupolar terms [36], the rotation of Neptune would produce 
strong harmonics and their multiples at the synodic rotation period (14.46 hr) of Neptune in the rest frame of Triton [36]. The large tilt of Triton's orbital plane relative to Neptune's equator $\left(156.8^{\circ}\right)$ also produces a strong harmonic at the orbital period (5.88 d) of Triton (Fig. 2). An internal ocean would produce an appreciable induced magnetic field [37] at these harmonics that could be detected from orbit or by a well-placed flyby $[38,39]$.

\section{Magnetic induction experiments are critical for future Triton missions to enable detection of the presence of a subsurface liquid layer.}

\section{Where Triton fits in the Search for Life}

The question we are asking ourselves today is whether or not there is life anywhere else in our solar system besides Earth. What conditions are required for life to appear and evolve? Do the oceans of moons of other planets provide habitable environments? The easiest place to search for life right now is Enceladus, helpfully spewing the contents of its subsurface ocean into space. Such an investigation can arguably be accomplished by a Discovery or New Frontiers mission.

If we do not find life in Enceladus' ocean we will ask why not? If we do find life we will ask where else? Are all oceans hospitable to life? Once Europa Clipper mission reconnaissance is under way we will know where to successfully send the next mission to Europa, asking the same questions.

Triton is the obvious next candidate to answer these questions - does life exist everywhere in the solar systems' oceans? Or only in some oceans? To address these questions the groundwork must be laid now. We must first ascertain with certainty whether or not Triton is an ocean world. We must then characterize the nature of its ocean and its accessibility - are cryovolcanic terrains and/or active plumes accessing it? Are there cracks/faults/conduits with contact to the ocean reaching the surface? Is organic material generated in the tenuous atmosphere reaching this potential subsurface ocean?

\section{To prepare, to invest in the answer to a question we know is coming, we must send a mission to study Triton in this decade.}

\section{Future Missions to Triton (and Neptune)}

Given that we know so little about Triton a single flyby would yield major scientific rewards as evidenced by New Horizons' flyby reconnaissance of Pluto and Charon. A flyby mission such as the Discovery mission Trident [40] could determine whether or not Triton has a liquid interior layer, i.e. whether or not Triton is an ocean world. A flyby mission could image the side not imaged by Voyager, terra incognito in the northern hemisphere, and study Triton's interior structure including a subsurface ocean, surface ices, atmosphere, the energy powering its geysers, and drivers for Triton's intense ionosphere. Simply by carrying an imaging spectrometer and mapping the composition of Triton's surface units a flyby mission would answer numerous fundamental questions about exotic cryovolcanic processes. A magnetometer would be capable of establishing the presence of an ocean from electromagnetic sounding.

A Flagship mission in orbit around Neptune would yield scientific return on the entire ice giant system as well as both broader and more in-depth investigations of Triton itself. An orbiter with multiple Triton flybys offers the opportunity to do comprehensive mapping of Triton's surface and volatile distribution, to carry a robust payload, sound the deep interior of Triton from electromagnetic sounding, and to study time domain variability of Triton's atmosphere, 
magnetosphere, and surface. Compiling observations from multiple flybys of Triton also enables comprehensive characterization of a putative subsurface ocean, necessary for ascertaining habitability. A Neptune system orbiter could answer Triton science questions, and is the key to unlocking our understanding of both ice giants and to making the next major advance in Kuiper Belt dwarf planet science.

The combination of the two missions is complementary because we can study the diurnal to decadal temporal elements of change in what is already known to be a dynamic environment. How much do Triton's ices migrate as the subsolar point moves steadily northward? Are there any surface changes as a result of cryovolcanism?

The final argument for a mission to Triton comes from the broader community, in the form of the OPAG Roadmap to Ocean Worlds Recommendation: Among the candidate ocean worlds in the solar system "Triton is deemed the highest priority target to address as part of an Ocean Worlds Program. This priority is given based on the extraordinary hints of activity shown by the Voyager spacecraft (e.g., plume activity; smooth, walled plains units; the cantaloupe terrain suggestive of convection) and the potential for ocean-driven activity given by Cassini results at Enceladus. Although the source of energy for Triton's activity remains unclear, all active bodies in the Solar System are driven by endogenic heat sources, and Triton's activity coupled with the young surface age makes investigation of an endogenic source important." [14].

Based on ROW's recommendation OPAG placed a Neptune flagship orbiter as its highest priority mission for the next decade [41].

\section{A mission to Triton answers the call to Explore Ocean Worlds.}

References: [1] Smith, B. et al. (1989) Science 246:1422. [2] Conrath, B. et al. (1989) Science 246:1454. [3] Broadfoot, L. et al. (1989) Science 246:1459. [4] Tyler, L. et al. (1989) Science, 246:1466. [5] Stern, S. A. et al. (2018) ARAA 56:357. [6] McKinnon, W. et al. (1995) in Neptune and Triton. [7] Agnor, C. and D. Hamilton (2006) Nature 441:192. [8] Quirico, E. et al. (1999) Icarus 139:159. [9] Grundy, W. et al. (2010) Icarus 205:594. [10] Cruikshank, D. et al. (1993) Science 261:742. [11] Stern, S.A. and W. McKinnon (2000) Ast J. 119:945. [12] Schenk, P. and K. Zahnle (2007) Icarus, 192:135. [13] Nimmo, F. and J. Spencer (2015) Icarus 246:2. [14] Hendrix, A. and T. Hurford (2019) Astrobio 19:1. [15] Croft, S. et al. (1995) in Neptune and Triton. [16] Prockter, L. et al. (2005). GRL 32:L14202. [17] Spencer, J. (1990) GRL 17:1769. [18] Hansen, C. and D. Paige (1992) Icarus 99:273. [19] Ingersoll, A. (1990) Nature 344:315. [20] Hansen, C. et al. (1990) Science 250:421. [21] Telfer, M. (2018) Science 360:992. [22] Elliot, J. (2000) Icarus 148:347. [23] Trafton, L. (1984) Icarus 58:312. [24] Holler, B. et al. (2016) Icarus 267:255. [25] Moore, J. and J. Spencer (1990) GRL 17:1757. [26] Soderblom, L. et al. (1990) Science, 250:410. [27] Kirk, R. L. et al. (1990) Science, 250:424. [28] Hansen, C. and R. Kirk (2015) LPSC 46, \#1832. [29] Belcher, J. et al. (1989) Science 246:1478. [30] Richardson, J. et al. (1995) in Neptune and Triton. [31] Stone, E. et al. (1989) Science 246:1489. [32] Krimigis, S. et al. (1989) Science 246:1483. [33] Mauk, B. et al. (1995) in Neptune and Triton. [34] Hussmann, H. et al. (2006) Icarus, 185:258. [35] Hammond, N. et al. (2018) JGR, 123:3105. [36] Connerney, J. et al. (1991) JGR-SP 96:19023. [37] Saur, J. et al. (2010) Space Sci. Rev. 152:391. [38] Nimmo, F. and R. Pappalardo (2016) JGR 121:1378. [39] Khurana K. et al. (2020), Manuscript in preparation for publication in Nature, Astronomy. [40] Prockter, L. et al., (2019) LPSC 50, \#3210. [41] Moore, J. and OPAG, Decadal Survey white paper, 2020. 